Voix et Images

\title{
En mémoire de l'avenir : les stratégies de transformation dans la narration de Jovette Marchessault
}

\section{Barbara Godard}

Volume 17, numéro 1 (49), automne 1991

Louky Bersianik

URI : https://id.erudit.org/iderudit/200945ar

DOI : https://doi.org/10.7202/200945ar

Aller au sommaire du numéro

Éditeur(s)

Université du Québec à Montréal

ISSN

0318-9201 (imprimé)

1705-933X (numérique)

Découvrir la revue

Citer cet article

Godard, B. (1991). En mémoire de l'avenir : les stratégies de transformation dans la narration de Jovette Marchessault. Voix et Images, 17(1), 100-115.

https://doi.org/10.7202/200945ar d'utilisation que vous pouvez consulter en ligne. 


\title{
En mémoire de l'avenir: les stratégies de transformation dans la narration de Jovette Marchessault
}

\author{
par Barbara Godard, université York
}

Je suis le Scribe! J'acquiers une main glorieuse et je relate. J'invente. Je transcris. Je dénature et j'altère. J'exagère. Jinterprète et je parle. J'écris, je m'accroche vigoureusement aux mots avec ma ventouse ventrale. Dans un mouvement hélicoïdal je saisis la pelure des mots; je les mets à nu et je les éventre [...] Je les façonne sur mon tour de potier et j'en crève les bulles d'air horizontales. Je façonne dains un mouvement circulaire la vase de ma vie, la matière du livre [...] Il me semble important de créer de nouvelles constellations de mots qui seront comme des comètes: après métamorphose les mots mineurs deviendront des mots majeurs. 1

Dans cette mise en abyme autoréférentielle, Jovette Marchessault élabore son projet narratif, la figuration d'un monde virtuel qui, en brouillant l'opération de la deixis et par conséquent la logique narrative - une épreuve de l'étranger ou verfremdungseffekt -, déplace les frontières établies entre le réel et la fiction. C'est une tentative de transformer le " monde" par la mise en place d'un appareil discursif qui interroge la représentation et expose les stratagèmes du récit afin de produire du nouveau ${ }^{2}$. En cela, les récits autobiographiques de

1 Jovette Marchessault, Comme une enfant de la terre 1. Le crachat solaire, Montréal, Leméac, 1975, p. 135-136.

2 Ces jeux de langage *investigatifs * qui caractérisent *le texte interrogatif * déstabilisent la position du sujet du savoir offert au lecteur. Catherine Belsey, Critical Practice, London, Methuen, 1980, p. 90-91. 
Marchessault participent à un projet utopique féministe tout comme l'Euguélionne et Picture Theory, des fictions de l'étrangeté, de la distanciation plutôt que des cuvres naturalistes ${ }^{3}$. En passant de la mimesis à la poïesis, ces fictions rendent insolite ce qui semble habituel et font prendre conscience de la construction du sens. La fiction trouble la vraisemblance du genre. Ainsi propose-t-elle de nouveaux modèles à l'intérieur desquels les femmes pourront s'inscrire.

Dans cet article, je tenterai de repérer les stratégies que Marchessault déploie pour élaborer des codes de l'irréel dans ses récits exploréens, surtout la litanie, l'anaphore, l'apostrophe et l'allégorie, m'occupant ainsi de l'avenir $d u$ passé ${ }^{4}$, du travail de la mémoire qui aboutit à un travail de mémorialiste pour produire des mémoires. L'allégoresis s'élabore dans un dédoublement du sujet qui se forme avec l'autre, l'absent rendu présent dans/par l'écriture, et forme une configuration. De la mémoire à travers l'écriture advient une sujète "nouvelle". Le projet romanesque est déplacé par un projet discursif où le sujet de l'énonciation s'articule au féminin. Il faudra donc envisager les problématiques conjointes de la subjectivité et de la politique de la représentation, soit la sexuation du discours $^{5}$. Bien que mon analyse se limite au premier tome de la trilogie Comme une enfant de la terre, c'est-à-dire au Crachat solaire, elle pourrait se généraliser aux autres volumes, la Mère des herbes et Des cailloux blancs pour les forêts obscures, qui font partie de ce Song of Myself ${ }^{6}$. Les trois livres se divisent en chants plutôt qu'en chapitres. Ils partagent aussi l'immense force créatrice de la mémoire?, une stratégie de narration par laquelle je me souviens, avec une précision hallucinatoire ${ }^{8}$. J'aborderai cette analyse à partir d'une approche narratologique.

3. Darko Suvin, Metamorphoses of Science Fiction: The Poetics and History of a Genre, New Haven, Yale University Press, 1979, p. 52-53.

4 Cette phrase est le titre d'une étude théorique sur l'utopie par Alexandre Cioranescu (Paris, 1972) citée dans la bibliographie de Darko Suvin, op. cit.

5 J'emploie ce néologisme pour traduire ce concept évơué en anglais par «gender». Bien qu'elles renvoient au genre grammatical, les connotations du mot dépassent largement le domaine du langage pour englober tout ce qui traite de la construction sociale de la différence sexuelle. Les théories féministes socialistes et sémiotiques, dont je m'inspire, soutiennent que la différence sexuelle n'est pas d'ordre biologique, mais produite par des codes inscrits dans des représentations véhiculées par des discours et des institutions. "Gender", le masculin et le féminin, s'oppose à « sex", le mâle et la femelle.

6 Ceci est le titre des cantiques épiques du poete américain Walt Whitman dont l'œuvre de Marchessault tire beaucoup d'éléments, notamment sa manière d'elargir le sens du *je * à un concept pluriel où le poète devient la voix(e) d'un nouvel être. I celebrate myself, and sing myself, I And what I assume you shall assume. I For every atom belonging to me as a good belongs to you. "Song of Myself", Leaves of Grass [1855], Cambridge, Mass., Riverside, 1959, p. 25.

7 Des cailloux blancs pour les forêts obscures, Montréal, Leméac, 1987, p. 71.

8 Ibid., p. 50. 
La problématique du genre a été une des voies les plus productives pour la critique féministe qui partage l'hypothèse de Fredric Jameson sur l'idéologie des formes littéraires, à savoir que les genres sont essentiellement des institutions littéraires ou des contrats sociaux entre un écrivain et un public spécifique, dont le rôle est d'établir le móde d'emploi propre à un objet culturel donné ${ }^{9}$. Les textes construisent des discours sur la sexuation, reproduisent des discours sexistes, conservateurs, et/ou confrontent ces discours. L'analyse s'intéresse aux normes génériques surtout en ce qu'elles encodent des idéologies sexistes et porte une attention plus grande aux récits où s'élabore le sens commun. Les récits, selon Jean-François Lyotard, se prononcent non seulement sur ce qui est vrai, mais aussi sur ce qui est juste ${ }^{10}$; leur prétention à la vérité est justifiée dans l'acte même de transmission, dans leur côté " performatif", c'est-à-dire dans leur force illocutoire ${ }^{11}$. Mais les divers types de récits sont « naturalisés * différemment selon les pratiques signifiantes et les discours hégémoniques. Chaque signe est un idéologème dont la forme est déterminée par l'échange des signes et par l'interaction des participants à cet échange. Quand les formes changent, le signe se modifie aussi. Cette conceptualisation d'un réseau oppositionnel des discours et des formes (des idéologèmes) permet de théoriser la sexuation des formes et des discours narratifs, productions matérialistes de signes par deux groupes sociaux différents, deux communautés d'interprétation. La narrativité fonctionne comme une * technology of gender * œuvrant dans un espace sémiotique, celle des représentations et des discours, un dispositif politique produisant des effets dans des corps, des comportements et des relations sociales ${ }^{12}$. Ainsi, elle ne se situe pas dans «une histoire», un contenu thématique, mais plutôt dans un réseau de relations par lesquelles le savoir est rendu compréhensible aux lecteurs et aux lectrices ${ }^{13}$. Selon la narratologie, la grammaire du récit nécessite une transformation: un état d'équilibre initial se modifie en un nouvel équilibre. Selon une logique d'opposition, l'état initial se transforme en un état contraire (le cru en cuit) ${ }^{14}$, par une opération déictique (ici/ ailleurs). La spécificité de la narration se trouve dans la manière dont une séquence temporelle est perçue comme une séquence causale.

9 Fredric Jameson, The Political Unconscious: Narrative as a Social Symbolic Act, Ithaca, Cornell University Press, 1981, p. 106. Ma traduction.

10 Jean-François Lyotard, The Postmodern Condition, translated by Geoffrey Bennington and Brian Massumi, Minneapolis, University of Minnesota Press, 1984, p. 31.

11 Teresa de Lauretis, Technology of Gender, Bloomington, Indiana University Press, 1987, p. 3.

12 Ibid., p. 41.

13 Anne Cranny-Francis, «The Women Men Don't See: Feminist Rewritings and Rereadings of Generic Fiction », présenté à ISSISSS 1987, Toronto, juin 1987, p. 8. Voir aussi Feminist Fictions, Londres, Frances Pinter, 1989.

14 Claude Lévi-Strauss, Structural Anthropology, Garden City, Doubleday, 1968; Algirdas-Julien Greimas, Du sens, Paris, Seuil, 1970. 
( « Alors* signifie à la fois * et» et * parce que *15.) Ainsi, le nouvel équilibre apparaît comme la conclusion logique et * naturelle * d'un processus temporel/transformationnel. Mais la mimésis temporelle masque l'opération de la causalité et le récit cache le fait qu'il a été construit par une communauté interprétative spécifique dont les intérêts sont avancés par la logique narrative elle-même. Ainsi, comme des féministes l'ont démontré, les contes de fées sont un dispositif du discours idéologique patriarcal efficace ${ }^{16}$.

L'impossibilité pour les personnages féminins, ou les femmesauteures, de s'inscrire dans un mythos autre que la narration d'une histoire d'amour a souvent été remarquée ${ }^{17}$. Cette histoire de mariage est réglée, comme l'a souligné Claude Lévi-Strauss, par une économie œdipienne d'échange des femmes entre le père et le fils ou entre le frère et le fils. La femme n'est qu'un signe d'échange dans le discours, une négativité (l'afemme) dans un discours hom $(m)_{0}$ sexuel ${ }^{18}$. L'ordre symbolique est réglé par un commerce des femmes. Le moteur même du récit est un cadavre de femme, l'absence - le manque initial - qui rend possible l'énonciation et la production du sens. Ainsi, une fonction camouflée du conte de fée et du roman est de délimiter l'espace social réservé aux femmes, leur rôle "naturel " dans l'espace qui leur est * propre », le rôle de la femmé meurtrie qui est ordonné par le contrat sacrificiel d'un ordre social et symbolique patriarcal.

C'est justement à ces normes discursives que Jovette Marchessault s'attaque en déjouant la logique temporelle du récit. Elle remet en question ce sacrifice des femmes et la violence de la rhétorique 19 qui la reproduit en refusant les oppositions du récit conventionnel avec sa clôture dans la mort (le mariage signifie la mort, selon ce mythos) pour arriver dans les dernières pages du Crachat solaire à la naissance d'une fille. Elle s'attaque aussi à l'illusion * naturaliste», ce qui l'entraîne à transgresser les codes, subvertir le monopole des hommes sur la possibilité de notre socialité, de notre culture. Le passage de la nature à la culture effectué par l'instauration de l'empire

15 Gerald Prince, A Grammar of Stories, The Hague, Mouton, 1973, et Shlomith Rimmon-Kenan, Narrative Fiction: Contemporary Poetics, Londres, Methuen, 1983, p. 19.

16 Marcia R. Lieberman, «Some Day My Prince Will Come: Female Acculturation through the Fairy Tales», College English, 34, decembre 1972, p. 383-395. Jennifer Waelti-Walters, Fairy Tales and the Female Imagination, Montréal, Eden, 1982.

17 Joanna Russ, *What Can a Heroine Do? Or Why Women Can't Write *, Images of Woman in Fiction, sous la direction de Susan Cornillon, Bowling Green, Bowling Green University Press, 1972, p. 3-20.

18. Luce Irigaray, Ce seze qui n'en eet pas un, Paris, Minuit, 1977, p. 163, 168.

19 Teresa de Lauretis, Technologies of Gender, Bloomington, Indiana University Press, 1987, p. 31-50. 
de l'hom $(m)_{0}$-sexualité dans sa médiation sociale établit les sociétés patriarcales comme des sociétés fonctionnant sur le mode du "semblant 220 .

Attaquer la représentation l'empêche de se dissimuler en faisant intervenir une parallaxe ou un déplacement de la perspective qui introduit l'hétérogène dans un discours du même sous la forme des protocoles de critique. Cette disjonction dans les normes discursives déstabilise les relations entre les systèmes de représentation et de signes. La représentation est alors perçue comme une productivité de sens. Pour enrayer l'ordre symbolique et sa logique de l'Être qui valorise le Même et le copula, Luce Irigaray préconise une logique du devenir hétérogène à cette économie de la représentation. C'est une logique de l'advenir-femme et non pas du devenir-femme, car elle ne postule pas la reproduction ni même la représentation, mais plutôt un déploiement stratégique du mimétisme, c'est-à-dire la deuxième sorte de la mimésis de Platon, la mimésis comme production.

À qui appartient le réel? Cette question pointe dé nouveau l'énonciation où sont fixés les "effets de réel». Car l'analyse de la représentation en tant que signe, processus de signification ou sémiose, nous fait envisager le fait que les représentations peuvent être "prises" comme signes qui "renvoient" à quelque chose d'autre, à une chose "réelle». La manière dont les signes sont pris pour quelque chose qui existe en dehors de la signification plutôt que pour des signes produits dans une certaine économie discursive est une problématique clé pour ces féministes. Quand il n'y a pas de "Vérité " unique, mais seulement des représentations ou des constructions différentes des vérités, certaines de ces représentations sont lues en tant que * faits" (le "réel»), d'autres en tant que «fiction ". Tout dépend de la manière dont elles sont conceptualisées, par qui, et dans l'intérêt de qui 21 . Comme le dit Nicole Brossard qui en fait la problématique centrale de son œuvre: La réalité des femmes n'est pas la réalité des hommes. ${ }^{22}$

Entre ces deux réalités s'érige la frontière entre la fiction et le réel. Les fictions des hommes, tel le système monétaire, sont des signes pris pour la réalité tandis que les réalités des femmes, telles que l'abus sexuel ou l'inceste, sont prises pour la fiction. Intercepter le réel devient alors un projet féministe, celui de réaligner les frontières entre la fiction et le réel. C'est un combat discursif mené par la fiction. L'écriture étant un outil qui permet de réfléchir les modes

20 Luce Irigaray, op. cit., p. 168.

21 Terry Threadgold, *Introduction *, Feminine/Masculine and Representation, sous la direction de Terry Threadgold et Anne Cranny-Francis, Sydney, Allen \& Unmin, 1990, p. 2-3.

22 Nicole Brossard, "Intercepter le rél», la Lettre aérienne, Montréal, les Éditions du Remue-ménage, 1985, p. 143. 
d'organisation de la pensée, le travail sur la perception virtuelle/ réelle se réalise dans la fiction, dans un lieu qui travaille la réalité, qui la mène à sa perte ${ }^{23}$. Les mots se chargent de la mémoire et ne ressemblent plus au réel: ils sont fictifs. Nous éprouvons alors un effet d'étrangeté qui nous fascine. ${ }^{24}$ Des féministes déploient la narration et les genres fortement codés et conventionnels d'une manière stratégique et autoréflexive pour "rendre visible* les discours conservateurs et sexistes qui y sont codés. Elles privilégient les genres tels le roman d'amour, le roman policier, la science-fiction et l'utopie, des genres de l'artifice, de l'étrangeté, plutôt que des genres réalistes, en dépit du fait que ces genres sont des plus conservateurs. Ces textes féministes manifestent le processus du conflit idéologique à l'intérieur de l'espace du texte même, dans une politique de la forme du genre. Traversant ainsi les frontières traditionnelles entre le réel et la fiction, esthétiquement, formellement, idéologiquement, ces écrivaines exposent l'existence de ces limites et proposent ainsi une position active et critique pour le lecteur/la lectrice du texte. On travaille le genre, en le fondant sur une logique de contradiction plutôt que sur une logique temporelle conventionnelle.

Certains de ces genres se prêtent plus facilement que d'autres à cette inflexion critique. Selon Darko Suvin, l'utopie est, comme la science-fiction, une méthode plutôt qu'un état, un projet d'expérimentation cognitive. C'est un jeu sérieux mené par quelqu'un qui a le sens des possibles autres que ceux de la nature, surtout des possibles parallèles. La méthode de l'utopiste peut se comparer à celle de l'approche hypothético-déductive des géométries topologiques, par exemple, en contraste avec des géométries euclidiennes 25 . La fonction de l'utopie est transgressive, comme le souligne Louis Marin: c'est une critique idéologique de l'idéologie ${ }^{26}$. La réalité prend la forme d'une figure discursive par un double jeu: d'une part, la projection métaphorique de la réalité * ailleurs", et d'autre part, le déplacement métonymique des structures de la société contemporaine. Lutopie interrompt le discours idéologique de la réalité historique actuelle en la représentant comme un système clos. On peut ainsi interroger les présuppositions en jeu dans ce processus de défamiliarisation. Le dialogisme de la figuration utopique construit par un discours fabulaire, mis en relation avec les représentations figées du discours idéologique régnant, simule et expose la contradiction. entre le réel et sa négation quand sa productivité est masquée par le

23 Ibid., p. 23.

24 Ibid., p. 145.

25 Darko Suvin, op. cit., p. 53.

26 Louis Marin, Utopics: The Semiological Play of Textual Spaces; traduit par Robert A. Volrath, Atlantic Highlands, N.J., Humanities Press International, 1984, p. 78, 195. 
discours idéologique. La figuration d'un temps et d'un espace discursifs nouveaux produit une structuration cognitive nouvelle qui réalisera une transformation sociale. Ainsi, la pratique de l'utopie avance un projet féministe de critique et de transformation des codes du genre romanesque en autant qu'ils se révèlent être des codes du discours sexué.

Marchessault déploie toutes les conventions de la distanciation utopienne dans le Crachat solaire, la juxtaposition de deux places et de deux temps: le voyage avec un guide ${ }^{27}$ dans un pays étranger (les États-Unis, le Mexique) et un temps éloigné (l'empremier, l'histoire de la colonisation de la Nouvelle-France), ici un déplacement et un transport par la rêverie et la mémoire; la société québécoise actuelle, le référent absent du texte, indiquée discursivement à travers la citation plutôt que signifiée dans des descriptions; la fonction de la figure utopienne pour l'analyse plutôt que pour la prédiction. Surtout, Marchessault déploie un style qui déplace les oppositions déictiques du maintenant et pas-encore par la litanie et l'apostrophe pour explorer le présent de l'énonciation comme une combinatoire des possibles hétérogènes et contradictoires. Ainsi, elle pose des indịces quant à l'action à entreprendre pour transformer l'avenir: la lutte politique les références à l'histoire des Québécois à la recherche de l'indépendance en 1837 (p. 176), les voix de libération, nous les survenants (p. 157) et la libération du cannibalisme européen (p. 155); je vais m'approprier la terre amérindienne, dans une frénésie de traces je vais lui imprimer la marque de mes roues, de mes pneus: me l'annexer! (p. 59-60) Mais ce sont surtout la lecture et l'écriture - la machine d̀ traverser le temps (p. 89) ${ }^{28}$ - qui transportent le sujet dans un ailleurs nouveau. [J]e n'étais qu'un lieu de passage pour ces livres qui circulent dans l'éther comme des comètes. A travers ces livres, je voyageais moi aussi sur un tapis d'étoiles... (p. 58) La lecture/ré/ écriture est l'advenir-femme, la production d'un sujet de l'énonciation au féminin. [J]e prenais des piles de notes sur l'androgyne primordial, j'écrivais en hauteur, en largeur, en marge, en exaltation, en euphorie (p. 58). Le voyage vers l'ailleurs, le monde parallèle critique de l'utopié, se réalise à travers des discours, des idéologèmes.

27 Tout comme il y a plus d'un temps et d'un espace, il y a plusieurs guides: Chere Francine, une des femmes telluriques posées par le sujet de l'énoncé; des écrivains tels Jack Kerouac, on the road, à travers l'Amérique du Nord, ou Malcolm Lowry au Mexique; mais surtout grand-mère, camarade de l'enfance et professeure de dessin, amérindienne et nomade.

28 La machine s'aiguise en tombant, en remontant d'un continent d l'autre, d'un corps a l'autre. S'aiguise au fil d'une hache, d'une epee d double tranchant. Et cela déchire des plafonds atmospheriques, des cauchemars crepusculaires, des toits de maisons, des draps de coton, vetements de fibres. Cela tranche des nouds, des réminiscences, des pressentiments, des idées éternelles, des tendances au concept. (p. 328) 
Je lisais donc des livres et déchiffrais des constructions mentales pour me libérer de mes anxiétés, lassitudes, angoisses. Je cherchais une idée pour parfaire ma vie même si je doutais de pouvoir y changer quelque chose en hauteur, profondeur et superficie rayonnante. Une idée! Rien de plus qu'une idée! Il y a dans l'idée un événement divin, prophétique qui se prolonge en nous. Je voulais le dépassement, jappelais la découverte du sang royal qui coule dans mes veines pour m'exalter et prendre le large avec mes tempêtes. Je réclamais la transfiguration. Rien de moins! (p. 125)

Le déplacement métaphorique en pays étranger est déconstruit, sa métaphoricité exposée, par un jeu métonymique. Les signes ne sont pas "pris " comme renvoyant à quelque chose d'autre, un "réelle" ailleurs, mais sont produits dans une économie discursive qui s'élabore dans l'acte de l'énonciation, dans l'écriture même. Il n'y a pas d'ailleurs, le texte nous informe, il n'y a que le discours, la route entre l'absence et l'espace, la sémiosis entre le vide et un référent.

Le Crachat solaire énumère des œuvres et des auteur(e)s qui ont pratiqué le "vécrire *, effectuant des transformations dans l'ordre symbolique afin de réaliser un changement social. L'exemple d'Alice Guy est instructif:

Veut faire plus, veut dire plus: elle ne veut pas seulement filmer, elle veut montrer l'esprit et le cour qui se libèrent du temps, qui se métamorphosent et tous les trajets, toutes les routes possibles entre l'absence et l'espace. Les fragments de vie sont des casse-tête qu'elle aime résoudre. Elle est prête à filmer à rebours s'il le faut. (p. 318)

Cette. Alice, la première femme réalisatrice de films au monde (p. 315), est l'héroïne de la grand-mère du sujet de l'énoncé, rendue présente dans la narration par le biais des mémoires d'Alice. Mais cela fonctionne comme promesse d'avenir, comme espoir pour ce sujet qui n'est pas encore venu au monde " réel ", mais qui voyage quelque part dans l'espace avant de choisir le lieu de son atterrissage, la famille où elle va naître. Alice a un père menuisier, une mère décédée... Pour elle la foi est une action, le doute une pensée. (p. 322) Son succès comme pionnière dans le cinéma est réalisé en dépit de son sexe et de sa classe. Ainsi, Alice est garante de l'avenir, de la transformation possible de la création artistique, pour ce sujet errant qui va choisir de "naître * dans la maison des ouvriers de souche autochtone: Moi, je ne suis pas encore là ! Je m'agite là-haut, à moitié vent, à moitié miracle, à moitié étincelle. Je m'en viens, on me prépare. (p. 322) S'écrire construit et maintient une vie:

Avec cette main glorieuse, j'écris avant que le cancer du doute et de la résignation ne dévore l'organe créateur, ne lui colle 
une étiquette de prix. Si un jour je cessais de m'écrire, de me peindre, la main glorieuse tomberait de mon corps comme une feuille morte. (p. 197)

De mémoire, le sujet de l'énoncé écrit en prolepse, c'est-à-dire en mémoire de l'avenir.

Ici se manifeste le travail de transformation critique. Le récit transforme le maintenant-temps-historique en récit de l'encore-àaccomplir ${ }^{29}$. Le point utopique se situe entre la carte ou les histoires officielles et les figures discursives, à un lieu d'interférence entre les représentations et le discours, entre l'espace et l'espace dù texte. L'écriture s'apprend en cheminant de lettre en lettre et la nature en cheminant de terre en terre... (p. 60) Ceci est le non-lieu, le lieu indéterminé, la figure "neutre" de l'utopie, ni ici, ni ailleurs mais entre. Ici "l'autre», "la négativité» du réel social et historique, surgit dans la figuration. ${ }^{30}$ Nous bafouillons le quotidien, écrit Marchessault (p. 185). Elle transforme la fonction du mundus inversus, le topos principal de l'utopie, par lequel est rendue explicite la critique du monde actuel. En ceci, l'écriture mobilise en même temps une image et son contraire ${ }^{31}$. Le récit ne résout pas cette contradiction; au contraire, il l'expose et la soutient en tant que discours, dans le conditionnel du désir, du fictif: Je voudrais être un pou! (p. 260) ${ }^{32}$

En effet, le récit de Marchessault amplifie la contradiction, car la logique temporelle du récit (alors, l'imparfait de l'énonciation) est déplacé par une logique de la contradiction (le paradoxe, le futur antérieur). Le voyage même est la contradiction (p. 59), à la fois un déplacement dans un ailleurs et l'arrêt dans une phrase. Tout est une affaire de grammaire, d'une logique de prédicats par où un sujet désirant se conjugue avec l'objet désiré pour compléter la syntaxe du récit.

Des champs de blé et de tomates de Marietta en Pennsylvanie au Chemin du Castor à Chertsey, Province de Québec. De la vallée au désert, du lac au terrain de stationnement: le voyage est pur acte de magie! Il est la force en action d'un pur désir. (p. 36)

Il' n'y a de changement d'un ici vers un ailleurs que dans la juxtaposition des mots, dans la contiguïté. Il n'y a plus de transport métapho-

29 Louis Marin, op. cit., p. 185.

30 Ibid., p. 196.

31 Darko Suvin, op. cit., p. 51.

32 On parlait faiblement, on parlait à voix basse, comme des convalescents en utilisant précautionneusement le futur des verbes avoir èt être. On avait le temps, on prenait son temps: la langue française est intarissable en temps de toutes sortes. On ne savait plus très bien les autres temps, les autres verbes, le point de départ de l'énoncé, le support de l'action parce qu'on était depuis trop longtemps le sujet de la Reine d'Angleterre. (p. 165) 
rique ou de progrès linéaire. Le récit tourne en rond pour terminer là où la lecture a démarré en prolepse avec la naissance anticipée du sujet de l'énoncé dans l'acte de l'énonciation. C'est une affaire de dédales (p. 115), de spirales (p. 92) et de signes cabalistiques (p. 114), des énigmes où le récit de l'aventure, du voyage, est absorbé par un récit herméneutique de lecture/ écriture. Les mots m'apparaissent comme des escaliers en spirales qui aboutissent à une porte. Qui souvre! (p. 105)

Bien que les deux mondes de la figure utopienne se trouvent ici; ce ne sont pas deux mondes séparés, mais deux mondes brouillés dans le présent de l'énonciation. Cela interrompt la grammaire de l'utopie qui se constitue dans un double récit, le récit d'un voyage ailleurs et le récit de la manière dont le narrateur le reçoit dans son propre pays ou temps. Les deux récits se rejoignent parce que le second est le lieu où les stratégies du premier - ses dislocations du point de vue, ses coutumes bizarres ou ses inversions du temps - sont naturalisées et justifiées. Il est aussi le récit qui propose une in(ter)vention sociale. Cet enchevêtrement des récits, l'un horizontal de l'action, l'autre vertical de l'herméneutique, constitue la vraisemblance du genre qui, dans l'utopie, oppose la vérité et la vraisemblance ${ }^{33}$. Mais en insistant sur la production et la réception d'un ailleurs dans l'écriture et la lecture, Marchessault défie ce vraisemblable du genre pour explorer les codes et les lois qui gouvernent la fiction et le "réelle». Son projet devient alors le dépistage des discours et des idéologies là où ils sont formulés dans la configuration du sujet.

Cette re/présentation qui met en scène la productivité du. déplacement insiste sur. le paradoxe, la contradiction produite par un déplacement métonymique dans la rencontre des airs froid-chaud.

Une cuiller à dessert par tasse d'eau polluée! Infuser un siècle ou deux et en prendre trois tasses par jour en relisant l'Acte de l'Amérique du Nord britannique sous des latitudes et longitudes où l'air qui circule toujours d'ouest en est, amène les. courants d'air froid de l'Arctique et les courants d'air chaud des Tropiques. (p. 189)

Le point de rencontre des contraires est la litanie, une énumération des lieux et des temps fort hétérogènes qui ne sont pas subordonnés à une structuration par une logique de prédicats. Le calendrier même n'est qu'une litanie de fêtes (p. 83). Le sujet de l'énoncé vole à travers le continent et dans le temps par la litanie des noms propres. Elle exauce son vœu de retrouver mes terres en se remémorant les noms des saintes et des héroïnes de la Nouvelle-France:

33 Tzvetan Todorov, The Poetics of Prose, traduit par Richard Howard, Ithaca, Cornell University Press, 1977, p. 86-87. Todorov analyse ici la vraisemblance du genre des romans défectifs. 
Quelle cavalcade! Des amazones à la poursuite d'un fugitif céleste avec à leur tête la très vénérable Marie-Marguerite du Frost de la Jemmerais, veuve d'Youville, fondatrice des Sours de la Charité de l'Hôpital Général de Montréal, dites sœurs Grises, Madeleine de Verchères, Jeanne Mance, Marie de l'Incarnation, Madame de la Peltrie, Madame d'Ailleboust, Isabelle Blondeau, Sœur Sainte-Hélène de la Passion, Sœur Sainte-Vitaline, la très pieuse Marguerite Bourgeois et ses compagnes Edmée Chastel, Catherine Crolo et Marie Raisin. Et c'était vrai!... Nous gardions sous nos robes noires un petit scapulaire... (p. 105)

Quelle cavalcade, en effet. L'espace aussi s'enjambe à grandes envolées, non par les pas géants dù grand lévrier, autobus de la compagnie Greyhound, mais par la magie de l'écriture, par la stratégie de la litanie qui $\mathrm{re} / \mathrm{c}(\mathrm{s})$ ite les grands voyages des explorateurs de l'Amérique:

Shippegan en octobre, Caraquet, Maisonnette, Restigouche: le muscle de pierre qui protège le cœur est abattu par le bélier du voyage. Nous nous rappelons les parcours de Jean Nicolet du côté du grand lac Michigan, ceux de Louis Jolliet du côté du Mississippi, de Boston, New York, sur des eaux pas encore encombrées, ceux d'Étienne Brâlé sur le lac Ontario, le lac Huron, ses portages autour de la baie Georgienne, de la baie Chesapeake... En novembre à Trois-Pistoles, Val-Brillant, Sayabec, Lac du Portage, Grand Métis, Cap à l'Orignal. Nos parcours et nos voyages de jadis nous ont scindés en de multiples morceaux! (p. 175, 177)

Dans cet emploi de la litanie qui ramène le temps de la narration constamment au temps présent de l'énonciation, Marchessault déplace, tout en conservant, le détail obsessif qui caractérise la figure utopique. Il n'y a plus d'arrêts avec de longs passages descriptifs par où on signifie le "réelle " de l'autre monde. Car Marchessault propose une transformation de la subjectivité. Ces litanies exposent les mécanismes discursifs de l'effet de réel en les rendant performatifs. Le récit de voyage produit une géographie par/dans l'inscription. Des noms sont écrits sur et dans un territoire qui est le point de référence pour le discours. L'addition d'un actant transforme la simultanéité en temps discursif ${ }^{34}$. Cependant, la remémoration de Marchessault enraye cette transformation en insistant sur l'aspect purement discursif de ce récit de voyage. La réalité n'est pas signifiée, mais indiquée discursivement par l'ostension. La récurrence toponymique est une substitution linguistique pour la simultanéité. Il n'y a que des 
noms de lieux, il n'y a que la lecture, l'écriture. Il n'y a pas de carte, il n'y a que du discours.

La poïesis s'installe dans ce jeu autoréférentiel où les noms propres ne se réferent qu'à eux-mêmes. Leur fonction générique de désignation, d'ostension, qui opère dans un système classificatoire, est ici sous rature, manifestant leur existence fictive. En tant que simulacre d'une 'émergence, les noms propres signifient que la fiction est une réalité, et que la réalité est une fiction ${ }^{36}$. La répétition exagérée de la litanie arrête le mouvement linéaire du récit et l'amène, par la redondance, à se fixer sur le signifiant. Cela aussi déplace l'effet de réel, la mimésis de l'utopie conventionnelle avec son alternance entre deux mondes, vers la poïesis de l'énumération discursive. Ici se redéploie l'équivoque de l'utopie, sa signification ambiguë comme le négatif d'un positif, oùtopos ou comme le positif d'un négatif, eù-topos. Cette équivoque du nom propre déstabilise les fondations mêmes du roman, fixées sur l'identité garantie par le nom propre, qui postule une existence en dehors des sèmes, une fonction cohésive établie dans le roman dès ses débuts au dix-huitième siècle ${ }^{36}$.

Cette ambiguïté s'attache à l'emploi exagéré des noms propres du pays abordé d'un autre angle, celui de la constitution d'un sujet de l'énonciation. Dans son analyse des * Noms du pays*, Julia Kristeva reprend l'hypothèse de Benveniste pour qui les toponymes sont des déictiques, c'est-à-dire des marques du discours, définis dans une instance d'énonciation spécifique, où ils fonctionnent pour mettre de la distance entre eux-mêmes et le sujet, le référent et les signes. Ils sont des modalités de spatialisation qui servent à distinguer deux espaces différents.

L'emploi des déictiques dans le développement du langage chez l'enfant se produit à la période de l'émergence d'un sujet, en conjonction avec le fort / da. Caractérisée par l'émergence des négations et des oppositions, l'emploi des références spatiales, des démonstratifs, des "topos" et des noms propres, cette période du développement de l'identité aboutit à la capacité d'autodésignation, * je-objet du discours *. Pour Kristeva, le nom propre est un substantif de référence, comme un démonstratif, mäis avec une signification indéterminée à cause de la position instable de l'identité du sujet de l'énonciation chez l'enfant ${ }^{37}$. Par conséquent, les noms propres du pays

35 Ibid., p. 90.

36 Ian Watt, The Rise of the Novel, cite dans Patricia Parker, *The (Self)-Identity of the Literary Text: Property, Propriety, Proper Place, and Proper Name in Wuthering Heights *, dans Identity of the Literary Text, sous la direction de Mario J. Valdes et Owen Miller, Toronto, University of Toronto Press, 1985, p. 92.

37 Julia Kristeva, «Place Names», dans Desire in Language: A Semiotic Approach to Literature and Art, sous la direction de Leon Roudiez, traduit par 
sont ambigus sémantiquement mais dynamiques. L'archéologie du topos nous amène, alors, à constater la nature équivoque du rapport sujet/objet dans le discours, rapport contradictoire qui est la problématique de la voyagerie dans le Crachat solaire: la quête du moi dans la rencontre de l'autre qui est le moteur même de tout récit de voyage ou d'ethnologie. Dans le récit de Marchessault, cette instabilité est productive et dynamique. Un univers de plénitude où la bataille du Moi et du Soi s'orchestrerait dans le dynamisme des contraires... (p. 206)

La thématisation de cette problématique de la construction du sujet de/dans l'énonciation se réalise dans le Crachat solaire par l'emploi de l'apostrophe, une figure de rhétorique par laquelle le sujet interpelle l'autre et se configure dans cette relation de l'altérité réciproque. Les autres apostrophés par le sujet de l'énonciation du Crachat solaire sont multiples. Cela commence avec le chien, le lévirier qui transporte le sujet de l'énoncé: [t]oujours plus au Sud mon chien! (p. 44) Il y a aussi la compagne de voyage, Francine, esprit tellurique qui, avec d'autres voix, est le "tu" ou le "vous" dont l'absence nécessite le travail de la mémoire. Tu n'es plus là, ma chère Francine... (p. 128) Vous étiez nos voix en Amérique. Toi ma chère Francine, mon cher Michel-le-Galopeur, ma chère Martine-la-Brûlure, ma chère Mariela-Rancune, mon cher Jean-Luc-Rapide-Danseur (p. 181). Il y a aussi les adresses à des personnages de livres ou à des auteurs: Ave Joe Beef! Ave Sigmund Freud! (p. 229) Ainsi, les catégories comprises dans la deuxième personne s'élargissent, deviennent hétérogènes. Dans le dernier chant, cela devient une voix cosmique et compréhensive qui englobe à la fois les personnages en souvenir, les caractères des légendes et des récits, les auteurs de ces personnages et les lecteurs du texte le Crachat solaire. Reliez-vous! Ralliez-vous! (p. 326) L'apostrophe revient comme un refrain, la répétition anaphorique convoquant le "vous" à des actions propices à la configuration des paroles et discours: Rappelez-vous! ralliez-vous! Éveillez-vous! Soyez les éveillés, les éveilleurs. (p. 327)

L'apostrophe à un absent mort ou sans voix est la figure de la prosopoeia, la figure dominante de l'autobiographie, selon Paul de Man 38. Cette fiction de la voix prend la forme d'une adresse à un absent qui postule la possibilité d'une réponse de l'autre. La trace de l'autre en nous - le dé-facement, l'effacement du visible - confère le pouvoir de la parole à l'autre, qui nous le rend. L'autre habite en

Thomas Cora, Alice Jardine et Leon S. Roudiez, New York, Columbia University Press, 1980, p. 290.

38 Paul de Man, Blindnes and Insight: The Rhetoric of Contemporary Criticism, $2^{e}$ édition revue, Minneapolis, University of Minnesota Press, 1983, p. 75-76. 
nous, entre nous et ainsi nous ne sommes jamais identiques à nousmêmes. La prosopoeia ou l'apostrophe est le trope de la lecture. L'allégorie de la lecture, selon de Man, concerne l'échange de faces et le défacement - figure, figuration et défiguration (p. 76). Dans le Crachat solaire, la différentiation d'un je/objet du discours, établie dans ce dialogue avec l'autre, se déstabilise. Le «je» se confond avec le "vous" dans le * nous *. De plus, le sujet de l'énonciation se mue en sujet de l'énoncé et en sujet parlé. Les " elles " se métamorphosent en "nous» 39 . Le «je " aussi se transforme en «nous* et parle du «.je* comme *elle». "Tu * se mue en *il " (p. 82). Les distinctions entre le référent et le discours s'effacent quand tout est discours et lecture. Le mouvement du récit pour réaliser la conjonction du sujet et de l'objet tourne en rond quand on ne peut plus les distinguer l'un de l'autre. Cette trace de l'autre est la finitude de la mémoire. Le travail de la mémoire produit une fiction de la prosopeia, une allégorie: la conjonction de la narration et de la mémoire produit un récit qui résiste ainsi à toute totalisation du genre épique.

Avec ce sujet hétérogène et dynamique, le récit nous renvoie au temps archaïque, à la lumière de l'Ancienne-des-temps (p. 93), pour préparer les nouvelles conditions d'énonciation, en réorganisant le politique dans une communauté sans division de races ou d'ethnies. L'invocation de "vous", d'une communauté de lecteurs, est la précondition de toute énonciation. Le récit tisse les liens entre *je " et * tu*. Le «nous", écrit Kristeva, est le lieu du contrat social des illusions, idéalisations, erreurs, constructions ${ }^{40}$. Ce "nous" hyperbole n'est pas isomorphe avec le «je ", car ce n'est qu'une formation temporaire des projections et des identifications qui règlent le changement des images par où le "nous * se métamorphose en collectif, se compose une histoire. Toute autobiographie aspire à produire une image collective et publique pour "vous" et pour "nous". Celle qui parle de "nous" n'est pas une analyse, mais une histoire qui s'analyse 41 . Ainsi, le Crachat solaire ne procède pas d'énigme en énigme, mais fait plutôt venir l'énigme comme condition d'énonciation. À partir de ce non-lieu, cet entre/antre, il y a une nouvelle vie qui se forme, un corps d'écriture qui n'est pas encore un corps écrit. Le Crachat solaire est l'écriture d'une naissance dont la fin est la condition de l'incipit. Le sujet de l'énoncé n'émerge que pour réciter [j]e suis d'origine céleste et je suis née à Montréal dans les années trente (p. 11), que parce que le temps.

39 Des amazones à la poursuite d'un fugitif céleste [...] la très pieuse Marguerite Bourgeois et ses compagnes Edmée Chastel, Catherine Crolo et Marie Raisin [...] Nous gardions sous nos robes noires [...] (p. 105)

40 Julia Kristeva, «My Memory's Hyperbole», The Female Autograph, sous la direction de Domna C. Stanton, Chicago, Chicago University Press, 1987, p. 220. C'est moi qui souligne.

41 Ibid. 
est venu, parce que la narration $a$ eu lieu. Le temps est venu, ils me font signe et je plonge, je plonge, je descends en flèche comme un crachat solaire (p. 348). Le processus de cette venue pour se re-citer est l'histoire qui s'analyse dans ce récit autoréférentiel.

Cette remontée dans le temps est la narration même qui est toujours le dédoublement: la re/citation, la répétition ou le travail de la mémoire. Un travail sur la trace d'ailleurs, qui ne pourrait s'exercer que dans la disjonction qui marque la différence entre deux moments, deux lieux. Parmi les procédés qui étirent le sens en fiction, les procédés hyperboliques de la mémoire du sujet au pluriel sont ceux qui favorisent l'émergence de l'histoire. Si pour Kristeva, c'est la mémoire qui est hyperbole, pour Paul de Man, c'est le vertige de l'hyperbole qui évoque le dédoublement et la simulation, des opérations du signe dans l'ironie et l'allégorie, deux tropes importants dans une rhétorique de la temporalité $\mathbf{4 2}$.

L'allégorie, qui vient du mot grec allegoria qui veut dire "parler autrement ", est un récit à deux niveaux de sens où l'autre parle dans le sujet qui parle. C'est une figure de la disjonction, qui marque la distance en relation avec sa propre origine ${ }^{43}$ : ses deux niveaux ne se fondent pas dans une union du sujet/objet comme dans le symbole. Le palimpseste de l'allégorie fonctionne par moyen de correspondances. Mais la découverte de l'analogie est une découverte qui, loin d'être spontanée, implique la discontinuité d'une renonciation 44 et d'une rupture entre le sujet et l'objet. Ainsi, l'allégorie est la figure de la temporalité; notre conception de l'histoire est un effet de l'allegoresis ${ }^{45}$. La rhétorique de la temporalité est la rhétorique de la mémoire, marquée par la discontinuité, l'hétérogénéité.

Si l'allégorie est disjonctive, une allégorie de la disjonction sera toujours une réflexivité disjointe, une allégorie de l'allégorie qui ne peut jamais, dans son autoréflexivité spéculaire, se rejoindre ellemême. Sa mémoire est alors une promesse de ré-collection qui ne peut pas se réaliser ${ }^{46}$. Car l'allégorie parle (à travers) la voix de l'autre, d'où son effet de re-venant, de spectre, d'où aussi sa disjonction asymbolique ${ }^{47}$. La mémoire est la voix de l'autre qui parle avant soi, qui signe à sa place, dans des citations - toujours de l'autre. C'est-à-dịre à la place de l'autre ${ }^{48}$. L'allegoresis est ainsi la figure de la lecture.

42 Paul de Man, op. cit., p. 215.

43 Ibid., p. 207.

44 Ibid., p. 205.

45 Jacques Derrida, «tThe Art of Mémoires », Memoires: For Paul de Man, traduction de Avital Ronelle et Eduardo Cadava, New York, Columbia University Press, 1986, p. 74.

46 Ibid.; p. 76.

47 Ibid., p. 80.

48 Ibid., p. 29. 
C'est dans et par l'allégorie de la lecture que Marchessault dédouble les niveaux du récit utopique. C'est par la lecture et la réc(s)itation de ses lectures qu'elle se souvient de l'histoire du Québec et de l'histoire de la vie de la grand-mère, Louisa. Les vies et légendes sont les revenants avec lesquels Marchessault fait son travail de mémoire pour conjurer l'avenir. La mémoire collective ou familiale, revenant dans des écrits ou des histoires orales, se mue dans la recitation en des mémoires du Québec et des peuples amérindiens. Ainsi, Marchessault configure une littérature pour situer son récit dans une relation dialogique.

La promesse des voix, surtout de la voix de la grand-mère, est de nous faire entrer dans un autre temps (p. 325) ${ }^{49}$. Quand je repense $\grave{a}$ la vie de grand-mère, je frémis. Je tremble! Je m'émerveille devant sa vie comme je m'émerveille devant le Grand Canyon ou les chutes Niagara. (p. 262) Dans la grand-mère, elle trouve des traces de l'androgyne primordial (p. 262) qui, en revenant, déplacerait le corps meurtri de la femme, l'absence sur laquelle repose le récit traditionnel. D'autre part, en conjurant la voix de la grand-mère par le travail de la mémoire, le récit fait vẹnir une nomade dont la dérive produit des ruptures et ainsi du changement: Grand-mère avance dans le sens de son cour: fait arc-en-ciel dans la Ford. (p. 292)

Le Crachat solaire est une fiction stratégique que Marchessault élabore à partir de la mémoire, une fiction de la disjonction et de la déstabilisation de l'identité. C'est une fiction où il n'y a plus de héros, plus de victime à immoler, plus d'intrigue. Ici se déploie le "Si je" fictif, le sujet femme contraint à l'invention dans chacune de ses paroles. Cela entame la réalité « sociologique" pour dissoudre le personnage et pour déjouer les impostures de l'anecdote quotidienne. Lidentité est comme une science-fiction de soi dans la pratique du texte. Le travail de la déconstruction du discours s'oriente vers l'insistance sur la productivité de tout discours, de toute énonciation. D'une part, Marchessault interroge qui parle, pour qui, en quelles conditions, pour valoriser la naissance plutôt que la mort d'une femme à l'origine de la narration. D'autre part, elle insiste sur le fait que la représentation est toujours une mise en scène, une lecture, le travail stratégique avec le revenant, une re-présentation. Le projet féministe de la déconstruction se combine avec un projet de déploiement stratégique des fictions pour transformer le "réelle", des fictions de l'advenir-femme.

49 Trois des douze chants du Crachat solaire et tous ceux de la Mère des herbes convoquent la voix de l'autre, grand-mère, génitrice et créatrice, le re-venant avec qui le sujet de l'énonciation se configure dans son récit. 